\title{
Dislocation Mechanisms During High Temperature Creep Experiments On MC2 Alloy
}

\author{
TOURATIER Fabienne ${ }^{1, a}$, VIGUIER Bernard ${ }^{1, b}$, SIRET Christophe ${ }^{1, c}$ \\ LESTERLIN Sandrine ${ }^{2, \mathrm{~d}}$ and ANDRIEU Eric ${ }^{1, \mathrm{e}}$ \\ ${ }^{1}$ Université de Toulouse, Institut Carnot CIRIMAT, INP/ENSIACET, \\ BP 44362, 4 allée Emile Monso 31432 Toulouse cedex 4, France \\ 2 TURBOMECA, avenue Joseph Szydlowski, 64511 Bordes Cedex, France \\ âFabienne.Touratier@eramet-aubertduval.com , bbernard.viguier@ensiacet.fr , \\ cchristophesiret@wanadoo.fr , 'Sandrine.Lesterlin@turbomeca.fr , eric.andrieu@ensiacet.fr,
}

Key words: single crystal superalloy, creep, dislocation, climb, diffusion.

\begin{abstract}
.
The creep behaviour of MC2 single crystal superalloy has been studied at $1150^{\circ} \mathrm{C} / 80 \mathrm{MPa}$, with an applied load along [001] axis. The resulting dislocation microstructures were examined by transmission electron microscopy. The occurrence of a[010] type dislocations (with a zero Schmid factor) within the ordered $\gamma^{\prime}$ precipitates is often observed. It is shown that those dislocations moved by a climb process, based on a mechanism involving two dislocation systems and vacancy exchanges, as proposed in the literature. We calculate the vacancy fluxes associated with such a mechanism and show that the vacancy transportation can be easily insured by a simple diffusion process. This calculation shows that the diffusion and climbing steps do not seem to be the creep rate controlling mechanisms for those situations in $\mathrm{MC} 2$ alloy.
\end{abstract}

\section{Introduction}

Nickel based superalloys are widely used in aeronautic industry as turbine blade materials. Indeed the blade are submitted to very severe conditions in terms of temperature and stress, which necessitates such a material with a very strong creep resistance. The unique properties of these single grained superalloys is due to the ordered $\gamma^{\prime}$ phase that precipitates in the solid solution $\gamma$ matrix. Many creep studies have been performed over the past decades in the temperature range up to $850^{\circ} \mathrm{C}$ with stress level that can reach up to $650 \mathrm{MPa}$ [1]. For higher creep temperatures the cuboidal precipitates of the ordered $\gamma^{\prime}$ phase coalesce in a anisotropic fashion to create "rafts" elongated in a direction normal ( $\mathrm{N}$ rafting) to the applied tensile stress for negative misfit alloys and parallel ( $\mathrm{P}$ rafting) for positive misfit (the reverse being true for compressive stress) [2]. More recently several creep studies have been performed in more severe situations, closer to the blade service conditions, and that may destabilize the $\gamma^{\prime}$ raft structure. Among these, one can mention oxidising conditions [3], non isothermal creep experiments [4, 5] and creep performed at very high temperature for which the raft microstructure may be destabilised either by morphological rearrangement [6] or by thermal dissolution of the ordered phase [7, 8]. Creep experiments performed at temperatures ranging from $950^{\circ} \mathrm{C}$ to $1250^{\circ} \mathrm{C}$ and relatively low stress (lower than 150 $\mathrm{MPa}$ ) have been reported during the last years together with detailed characterisation of the crept microstructures [9-12]. It has been recognised in all those studies that a macroscopic strain of the rafted microstructure necessitates some cutting of the $\gamma^{\prime}$ particles. In particular the role of a[010] dislocations moving through $\gamma^{\prime}$ rafts by some climb (or climb-assisted) mechanism was highlighted. Their motion recovers the microstructure in two instances : i) a[010] dislocations can annihilate $\gamma$ dislocations in the opposite channel and ii) they consume vacancies that are produced by climbing interfacial dislocations. The proposed mechanism for the climb of these $\mathrm{a}[010]$ (that can not feel the 
stress applied along [001] direction) consist on an exchange of vacancies between two dislocation systems. In the present paper we propose an estimation of the rate of vacancies diffusion and dislocation climb velocities during creep at high temperature in order to determine if those processes may be rate controlling. The flux of vacancies is calculated from the deformation rate in the one hand and from diffusion data on the other hand.

\section{Experimental details}

The material of this study was taken from a single crystal plate of the nickel-based superalloy MC2 (nominal composition in wt. \% Ni-7.2Cr-8Co-2Mo-7W-5.5Al-1.3Ti-5.6Ta). High temperature creep tests have been conducted at $1150^{\circ} \mathrm{C} / 80 \mathrm{MPa}$ along the [001] direction under laboratory air and constant load conditions. Creep tests were performed either in isothermal conditions or by cycling the imposed temperature between $1150^{\circ} \mathrm{C}$ and about $300^{\circ} \mathrm{C}$, holding time at $1150^{\circ} \mathrm{C}$ was in that case 30 minutes. Details on creep test conditions and apparatus can be found in [13]. After creep tests, specimens were mechanically grinded down to $1 \mu \mathrm{m}$ diamond paste and finally etched (using a solution of $13 \% \mathrm{H}_{3} \mathrm{PO}_{4}, 45 \% \mathrm{H}_{2} \mathrm{SO}_{4}$ and $42 \% \mathrm{HNO}_{3}$ under $3 \mathrm{~V}$ for $\gamma$ ' phase dissolution) allowing for scanning electron microscopy (SEM) observations. Quantitative data on the microstructure evolution have been measured as reported elsewhere [13]. Thin foils for transmission electron microscopy (TEM) were extracted from thin wall creep samples by means of a diamond saw as squares of edge $2.1 \mathrm{~mm}$ and thickness $200 \mu \mathrm{m}$. These slices were then mechanically polished down to about $50 \mu \mathrm{m}$ while tilted in order to achieve an orientation of the slice close to $(\overline{1} 21)$. The foils were then dimpled to about $20 \mu \mathrm{m}$ and ion milled using a Gatan PIPS ${ }^{\circledR}$ system. TEM observations and dislocations indexation were achieved in two-beam diffraction contrast on a Jeol 2010 operating at $200 \mathrm{kV}$ at the TEMSCAN Service of the University Paul Sabatier in Toulouse.
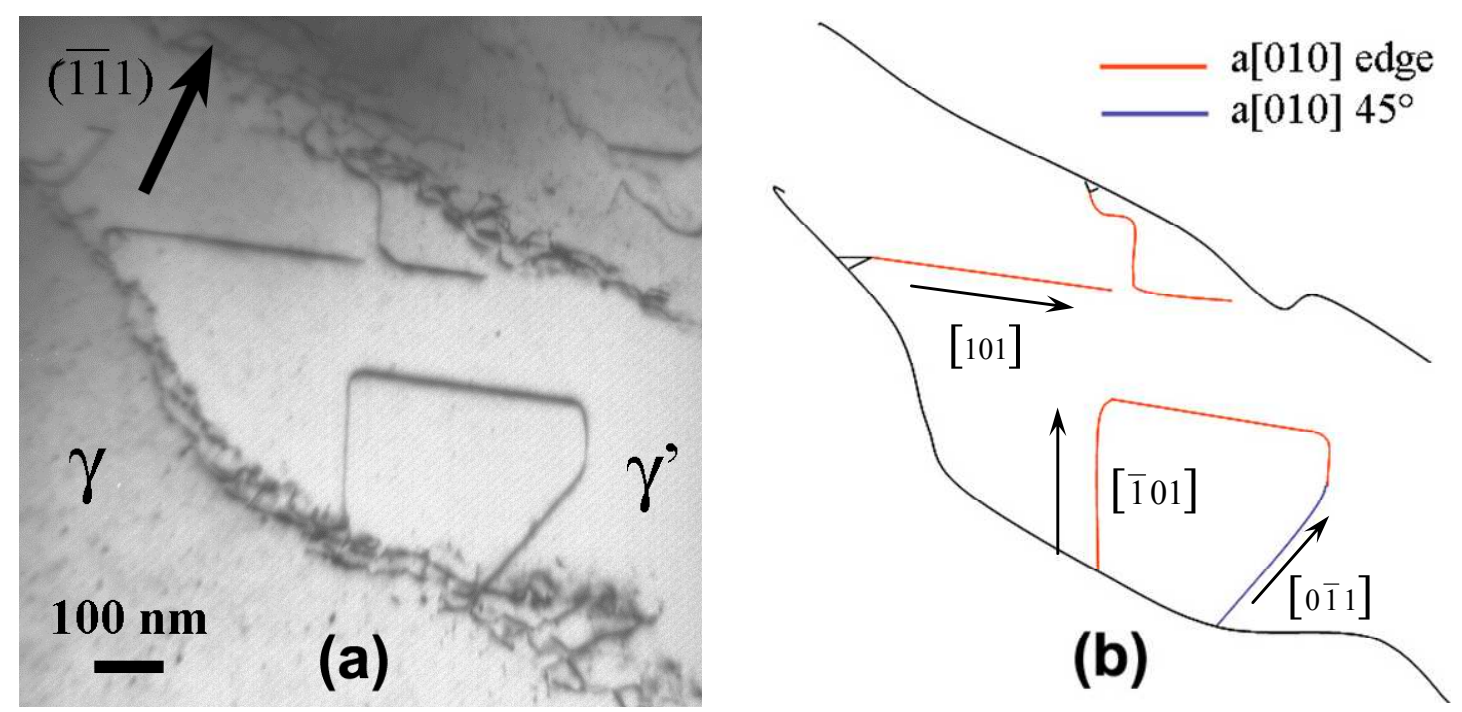

Figure 1 : Dislocation microstructure after creep experiment under thermal cyclic conditions $\left(1150^{\circ} \mathrm{C} / 80 \mathrm{MPa}\right)$. The bright field image in (a) is schematically represented in (b) with the indication of dislocation line directions. 


\section{Results and discussion}

Dislocations microsctructure and climb mechanism. We have focused our TEM dislocation observations within the $\gamma^{\prime}$ phase. Indeed during high temperature creep the superalloy microstructure evolves at the beginning of creep strain to form a rafted microstructure [8]. The rafts that are normal to the [001] load direction act as a barrier to matrix dislocations. A large scale creep strain is thus only possible if some deformation mechanism of the rafts operates [12, 14]. Fig. 1 presents a typical landscape of dislocations within a $\gamma^{\prime}$ raft after high temperature creep. This figure was obtained on a specimen that was tested on thermal cyclic creep experiment with the high temperature of $1150^{\circ} \mathrm{C}$ and 30 minutes holding time. Similar microstructures were repeatedly observed after high temperature creep in either isothermal or thermal cyclic conditions.

The bright field image in Fig. 1.a is taken with ( $\overline{1} \overline{1} 1)$ diffraction vector, the corresponding dislocation analysis is depicted in the scheme of Fig. 1.b. The complete contrast analysis of these dislocations required the use of several diffraction conditions in order to take into account the strong residual contrast effect under classical $g . b=0$ extinction conditions as was shown in $[15,16]$. The dislocations seen in the raft in Fig. 1 all have a[010] Burgers vector and their line is parallel to $<101>$ type directions, that is they are $45^{\circ}$ and edge dislocations segments. A more systematic study of dislocations after creep under various conditions showed that such dislocations are often seen in $\gamma^{\prime}$ rafts after creep at $950^{\circ} \mathrm{C}$ and that they are the vast majority of dislocations after higher temperature creep [17]. The morphology of the dislocations in Fig. 1.b shows that their configuration involved some climb mechanism. For instance the loop in the bottom of the raft in Fig. 1 presents line segments parallel to $[101]$ and $[10 \overline{1}]$ thus expends, at least partially, in the (010) plane, a pure climb plane for a[010] dislocation.

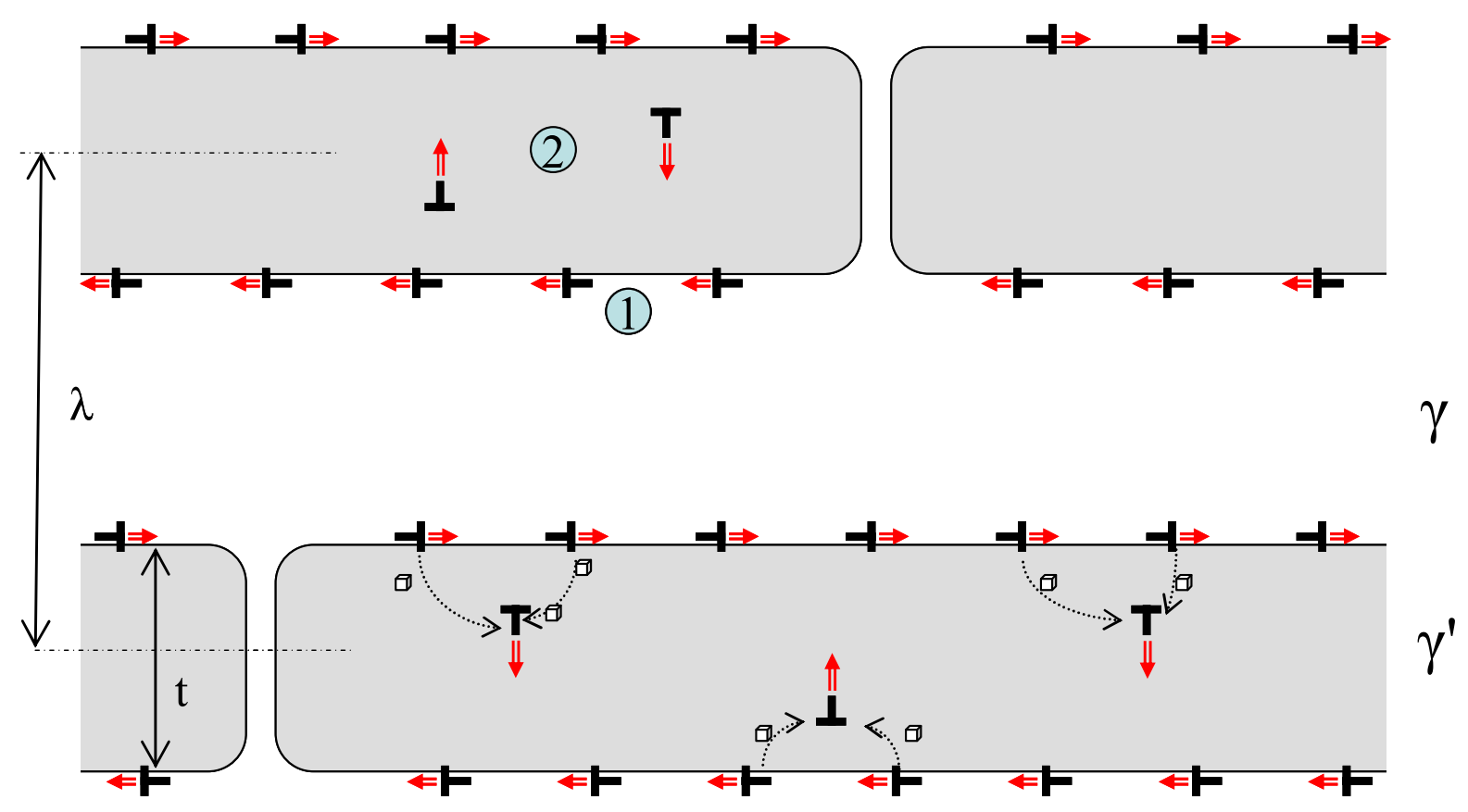

Figure 2 : Schematic of the superalloy microstructure during creep at high temperature showing the characteristics length $t$ and $\lambda$, as well as the two dislocation systems (1) and (2) moving by climb mechanism (double arrow) and the corresponding vacancies fluxes (dash line arrows in lower raft). 
The activation of $\mathrm{a}[010]$ dislocations have been questioned in the literature since, when creep load is imposed along [001] direction, they do not experience any resolved force (neither glide nor climb) from the applied stress. It is thus believed that their activation is a secondary process which acts as a recovery mechanism for the primary system which is actually driven by the applied stress and provides creep strain along [001]. Such recovery processes include i) the annihilation of $\gamma / \gamma^{\prime}$ interface dislocations when the a[010] dislocations have crossed the raft [10] and ii) the absorption of vacancies emitted by the climb of a dislocation of the primary system in the interface. Epishin and Link [9] have first shown that such a mechanism is involved in the high temperature creep strain of CMSX-4 and CMSX-10 superalloys. The creep strain in the direction of the applied stress is shown to be due to the transverse glide-climb of $a / 2<011>$ dislocations, producing vacancies. Those excess vacancies can be annihilated on pores and/or on a[010] dislocations, and thus act as a driving force for the climbing of these latter dislocations in $\gamma^{\prime}$ rafts. In turn the crossing of rafts by a[010] allows the annihilation of interface dislocations and also recovers the first straining system. Similar mechanism, involving two dislocation systems and a vacancy exchange has been demonstrated to operate during compression test of AlPdMn quasicrystals [18, 19]. The TEM observations reported here above show that a similar mechanism must operate in MC2 superalloy single crystal during high temperature creep along [001] axis.

This mechanism with the two dislocation systems is schematically shown in Fig. 2. For the sake of clarity in Fig. 2 only the climbing part of interfacial dislocations (system 1) has been shown. These interfacial dislocations could be either a/ $2<011>$ type moving by a combined glide - climb process [9] or dislocations with Burgers vector along the load axis [001] moving by a pure climb mechanism [20].

Climb velocity and diffusion rate. As discussed above, the proposed climb mechanism involves a vacancies exchange between two dislocations systems : system (1) which is located at the $\gamma / \gamma^{\prime}$ interface, is driven by the applied stress and produces vacancies ; and system (2) within the $\gamma^{\prime}$ raft, which crosses the raft by absorbing vacancies, thus relaxing mechanical state - indeed this crossing shrinks the specimen in transverse direction and accommodates the macroscopic deformation - and also permits to annihilate matrix dislocations. The goal of the present calculation is to check if the vacancies flux between dislocation systems (1) and (2) is rate limiting or on the contrary is fast enough as regard to the macroscopic plastic strain. In other words and in a more general instance, the question is to determine if climb velocity may control the creep rate or not.

During a steady state creep deformation, all the vacancies created by the climb motion of system (1) dislocations must be transported to sinks for being annihilated. Lets consider a macroscopic strain rate $\dot{\varepsilon}_{\text {climb }}$ created along the [001] direction by a density $\rho$ of interfacial dislocations climbing in the (001) interfacial plane at the velocity $\mathrm{v}_{\mathrm{climb}}$ :

$$
\dot{\varepsilon}_{c \operatorname{limb}}=\rho b_{[001]} v_{c \lim b},
$$

in which $b_{[001]}$ is the component of the Burgers vector along [001] (climbing part of the dislocation). The density can be expressed using the total length of dislocation lines L over a given surface of raft S and $\lambda$ the period of the $\gamma / \gamma^{\prime}$ structure as defined in Fig. 2, so that the Orowan law can be rewritten :

$$
\dot{\varepsilon}_{c \text { limb }}=\frac{L}{S(\lambda / 2)} b_{[001]} v_{c \lim b} \text {. }
$$

The quantity at numerator in equation (2) represents the volume of matter displaced per unit time during the climb motion. That is the number of vacancies created by unit time $\mathrm{dN} / \mathrm{dt}$ multiplied by the atomic volume $\Omega$ :

$$
L b_{[001]} v_{c \lim b}=\frac{d N}{d t} \Omega \text {. }
$$


Since all those vacancies must be removed a vacancy flow due to climb $\Phi_{\text {climb }}$ is established through the raft surface $\mathrm{S}$ :

$$
\Phi_{\mathrm{climb}}=\frac{1}{\mathrm{~S}} \frac{\mathrm{dN}}{\mathrm{dt}}=\frac{1}{\mathrm{~S}} \frac{\mathrm{Lb}_{[001]} \mathrm{v}_{\mathrm{climb}}}{\Omega}=\frac{\lambda}{2 \Omega} \dot{\varepsilon}_{\mathrm{climb}} .
$$

The vacancies flux can be estimated by using the microstructural and mechanical data, after creep experiments at $1150^{\circ} \mathrm{C}$. The atomic volume $\Omega$ is taken as $\mathrm{a}^{3} / 4$ where a is the cell parameter of $\gamma^{\prime}$ phase $(\mathrm{a} \approx 0.355 \mathrm{~nm})$. The period $\lambda$ was measured on the rapidly cooled specimen after creep tests interrupted for approximately half the life time at $1150^{\circ} \mathrm{C}(\lambda \approx 800 \mathrm{~nm})$ [13]. As a first approximation we have considered the creep rate associated to the climb mechanism equal to the macroscopic creep rate. This results in an over estimation of the vacancy flux since a part of the macroscopic strain is still due to some glide process in the matrix. We took an arbitrary reasonable (over-)estimated strain rate as $\dot{\varepsilon}_{\text {climb }} \approx 10^{-6} \mathrm{~s}^{-1}$. The resulting vacancies flux is obtained for a creep test performed at $1150^{\circ} \mathrm{C} / 80 \mathrm{MPa}: \Phi_{c \lim b} \approx 3.510^{16} \mathrm{vac} \cdot \mathrm{m}^{-2} \cdot \mathrm{s}^{-1}$.

Those vacancies produced at dislocation system (1) must be removed. The driving force for this removal through a diffusional mechanism is simply the vacancy gradient created by the applied stress. Indeed equilibrium between the mechanical and osmotic climb forces on system (1) fixes $\mathrm{C}_{\mathrm{i}}$ the vacancy concentration at the interface (considering the thermal equilibrium vacancies concentration $\left.\mathrm{C}_{0}\right)[21]$ :

$$
C_{i}=C_{0} \exp \left(\frac{\sigma \Omega}{k T}\right) \text {. }
$$

If one considers that vacancy sinks are far by the distance $\mathrm{d}$ and correspond to the thermal equilibrium vacancies concentration $\mathrm{C}_{0}$, the diffusional flow of vacancies can be estimated :

$$
\Phi_{\text {diff }}=D_{V} \frac{\left(C_{i}-C_{0}\right)}{d}
$$

where $D_{v}$ is the vacancy diffusion coefficient. The equilibrium vacancy concentration $X_{0}$ was estimated from enthalpy and entropy data extracted from the literature $[22,23]$ leading to $610^{-6}$ vacancies per atomic sites at $1150^{\circ} \mathrm{C}$. This value is very close to the one estimated by the VASP method [24]. The corresponding vacancies volume concentration is : $\mathrm{C}_{0} \approx 5.310^{23} \mathrm{~m}^{-3}$. The vacancy diffusion coefficient was estimated from the migration energy of a nickel vacancy on the nickel sites $[25,26]$. This gives values of about $\mathrm{D}_{\mathrm{v}} \approx 310^{-9} \mathrm{~m}^{2} \cdot \mathrm{s}^{-1}$ at $1150^{\circ} \mathrm{C}$.

The diffusion flux of vacancies is thus obtained by combining equations (5) and (6) and was calculated for a creep test at $1150^{\circ} \mathrm{C} / 80 \mathrm{MPa}$. If one consider that the dislocation system (2) is the vacancy sink then $\mathrm{d}=\mathrm{t} / 2=200 \mathrm{~nm}$ and one finds $\Phi_{\text {diff_disloc }} \approx 3.710^{20}$ vac. $\mathrm{m}^{-2} \cdot \mathrm{s}^{-1}$. One can see that this vacancies flux, generated by the diffusion process, is much larger (four order of magnitude) than the flux produced by the climbing mechanism. This means that the diffusion process is much faster than needed to eliminate vacancies at dislocations acting as sinks within the precipitates.

Alternatively one can calculate the flux for transporting the vacancies to the casting porosities in the single crystal, a typical diffusion distance would be $\mathrm{d} \approx 500 \mu \mathrm{m}$, which leads to a vacancy flux : $\Phi_{\text {diff_pore }} \approx 1.410^{17}$ vac. $\mathrm{m}^{-2} \cdot \mathrm{s}^{-1}$. This last value is still over the flux produced by the climb of interfacial dislocations. However it is now of nearly the same order of magnitude, indicating that diffusion of vacancies to porosities is a possible mechanism for eliminating them but this process might be rate limiting. This road is shown to be three order of magnitude less efficient than the diffusion to dislocations.

It is thus shown that provide there exists, within the rafts, dislocations that can serve as sink for vacancies, the diffusion process is more than sufficient for the vacancy exchange between the two dislocations systems (1) and (2). That means that diffusion and more generally climb process itself would certainly not be the rate controlling mechanism during high temperature creep of nickel base superalloys. We may show in a forthcoming paper [17] that the number of sinks, that is the density of $\mathrm{a}[010]$ dislocations in the ordered phase, is a more crucial point. 


\section{Summary}

Creep experiments have been performed under isothermal and cyclic thermal conditions at $1150^{\circ} \mathrm{C}$ under $80 \mathrm{MPa}$. Transmission electron microscopy observations reveal the presence of numerous dislocations with a [010] Burgers vector within the $\gamma^{\prime}$ rafts. It is shown that those dislocations move by climb process which is driven by the absorption of vacancies emitted by climbing interfacial dislocations. Calculations show that diffusion kinetics is fast enough to insure transport of vacancies from the emitted dislocation system to the system acting as a sink for vacancies $(a<100>$ dislocations climbing in $\gamma^{\prime}$ rafts and - to a lesser extent - pores). The vacancy diffusion (or climbing) process does not appear to be a rate controlling mechanism.

\section{References}

[1] F.R.N. Nabarro and H.L. De Villiers : The physics of creep, (Taylor \& Francis, London 1995).

[2] M. Veron, Y. Brechet and F. Louchet: Acta Materialia, 44 (1996), p. 3633.

[3] S. Dryepondt, D. Monceau, F. Crabos and E. Andrieu: Acta Materialia, 53 (2005), p. 4199.

[4] A. Raffaitin, D. Monceau, F. Crabos and E. Andrieu: Scripta Materialia, 56 (2007), p. 277.

[5] J. Cormier, X. Milhet and J. Mendez: Acta Materialia, 55 (2007), p. 6250.

[6] F. Touratier, E. Andrieu, D. Poquillon and B. Viguier: Mater. Sci. and Eng., A 510-511 (2009), p. 244.

[7] J. Cormier, X. Milhet and J. Mendez: Journal of Materials Science, 42 (2007), p. 7780.

[8] C. Siret: PhD thesis, Institut National Polytechnique Université de Toulouse (2010).

[9] A. Epishin and T. Link: Philosophical Magazine, 84 (2004), p. 1979.

[10] P.M. Sarosi, R. Srinivasan, G.F. Eggeler, M.V. Nathal and M.J. Mills: Acta Materialia, 55 (2007), p. 2509.

[11] A. Kostka, G. Malzer, G. Eggeler, A. Dlouhy, S. Reese and T. Mack: Journal of Materials Science 42 (2007), p. 3951.

[12] L.J. Carroll, Q. Feng and T.M. Pollock: Metallurgical and Materials Transactions A, 39 (2008), p. 1290.

[13] F. Touratier: PhD thesis, Institut National Polytechnique, Université de Toulouse (2008).

[14] R. Srinivasan, G.F. Eggeler and M.J. Mills: Acta Materialia, 48 (2000), p. 4867.

[15] A. Dlouhy, R. Schäublin and G. Eggeler: Scripta Materialia, 39 (1998), p. 1325.

[16] T. Link, A. Epishin, M. Klaus, U. Brückner and A. Reznicek: Materials Science and Engineering: A, 405 (2005), p. 254.

[17] B. Viguier, F. Touratier and E. Andrieu, (2010) to be published.

[18] F. Monpiou, L. Bresson, P. Cordier and D. Caillard: Philosophical Magazine, 83 (2003), p. 3133.

[19] F. Mompiou and D. Caillard: Acta Materialia, 56 (2008), p. 2262.

[20] F. Mompiou and D. Caillard: Materials Science and Engineering A, 483 - 484 (2008), p. 143.

[21] D. Caillard and J.L. Martin : Thermally Activated Mechanisms in Crystal Plasticity (Elsevier, Oxford 2003).

[22] G. Dlubek, O. Brümmer and N. Meyendorf: Physica Status Solidi, 39 (1977), p. K95.

[23] H. Numakura, T. Ikeda, H. Nakajima and M. Koiwa: Materials Science and Engineering A, 312 (2001), p. 109.

[24] X. Zhang and C. Y. Wang: Acta Materialia, 57 (2008), p 224.

[25] G.E. Murch and I.V. Belova: Journal of Materials Processing Technology, 118 (2001), p. 82.

[26] E. Partyka and R. Kozubski: Intermetallics, 12 (2004), p. 213. 